\title{
Noninterpolatory Integration Rules for Cauchy Principal Value Integrals
}

\author{
By P. Rabinowitz*and D. S. Lubinsky** \\ Dedicated to the memory of Peter Henrici
}

\begin{abstract}
Let $w(x)$ be an admissible weight on $[-1,1]$ and let $\left\{p_{n}(x)\right\}_{0}^{\infty}$ be its associated sequence of orthonormal polynomials. We study the convergence of noninterpolatory integration rules for approximating Cauchy principal value integrals
\end{abstract}

$$
I(f ; \lambda):=\int_{-1}^{1} w(x) \frac{f(x)}{x-\lambda} d x, \quad \lambda \in(-1,1) .
$$

This requires investigation of the convergence of the expansion

$$
I(f ; \lambda) \sim \sum_{k=0}^{\infty}\left(f, p_{k}\right) q_{k}(\lambda), \quad \lambda \in(-1,1),
$$

in terms of the functions of the second kind $\left\{q_{k}(\lambda)\right\}_{0}^{\infty}$ associated with $w$, where

$$
\begin{aligned}
\left(f, p_{k}\right):=\int_{-1}^{1} w(x) f(x) p_{k}(x) d x \quad \text { and } \quad q_{k}(\lambda):=\int_{-1}^{1} w(x) \frac{p_{k}(x)}{x-\lambda} d x \\
k=0,1,2, \ldots, \lambda \in(-1,1)
\end{aligned}
$$

1. Introduction. In the third volume of his monumental work, Applied and Computational Complex Analysis, Henrici [8, pp. 139-142] gave an algorithm for the numerical evaluation of Cauchy principal value (CPV) integrals. This algorithm was presented in a more explicit form in a recent paper, by one of the authors [15]. In neither case were convergence questions considered. In this paper, we shall analyze the convergence questions arising from the use of this algorithm.

Consider the CPV integral of the form

$$
I(f ; \lambda):=f_{-1}^{1} w(x) \frac{f(x)}{x-\lambda} d x, \quad-1<\lambda<1,
$$

where $w$ is an admissible weight function, $w \in \mathscr{A}$, that is, $w(x)$ is nonnegative and integrable in $[-1,1]$ and

$$
m_{0}:=\int_{-1}^{1} w(x) d x>0
$$

Received November 16, 1987.

1980 Mathematics Subject Classification (1985 Revision). Primary 65D30; Secondary 65D32, $41 \mathrm{~A} 55$

Key words and phrases. Cauchy principal values, numerical integration, noninterpolatory integration rules, orthogonal polynomials, functions of the second kind.

* Research completed during a visit to N.R.I.M.S.

** Part-time at the Department of Mathematics, University of the Witwatersrand, P.O. Wits 2050, Republic of South Africa. 
For such $w$, there exist sequences of orthonormal polynomials

$$
\left\{p_{n}(x):=p_{n}(w, x):=k_{n} x^{n}+\cdots, k_{n}>0\right\},
$$

with respect to the inner product

$$
(f, g):=\int_{-1}^{1} w(x) f(x) g(x) d x
$$

satisfying a three-term recurrence relation

$$
x p_{n}(x)=\alpha_{n+1} p_{n+1}(x)+\beta_{n+1} p_{n}(x)+\alpha_{n} p_{n-1}(x), \quad n=0,1,2, \ldots,
$$

where

$$
\begin{gathered}
\alpha_{n}:=k_{n-1} / k_{n}, \quad n \geq 1 ; \quad \beta_{n+1}:=\left(x p_{n}, p_{n}\right), \quad n \geq 0, \\
p_{-1}(x) \equiv 0 \quad \text { and } \quad p_{0}(x) \equiv k_{0}=m_{0}^{-1 / 2} .
\end{gathered}
$$

If we define $q_{n}(\lambda)$, the function of the second kind, by

$$
q_{n}(\lambda):=q_{n}(w, \lambda):=I\left(p_{n} ; \lambda\right):=f_{-1}^{1} w(x) \frac{p_{n}(x)}{x-\lambda} d x, \quad-1<\lambda<1,
$$

then the $q_{n}(\lambda)$ satisfy the same recurrence relation as the $\left\{p_{n}(x)\right\}$, namely

$$
\lambda q_{n}(\lambda)=\alpha_{n+1} q_{n+1}(\lambda)+\beta_{n+1} q_{n}(\lambda)+\alpha_{n} q_{n-1}(\lambda), \quad n=0,1,2, \ldots,
$$

with starting values $q_{-1}(\lambda) \equiv-1, q_{0}(\lambda) \equiv I\left(p_{0} ; \lambda\right)$ and $\alpha_{0}:=m_{0}^{1 / 2}$. If we denote by

$$
a_{k}:=\left(f, p_{k}\right)
$$

the Fourier coefficient of $p_{k}(x)$ in the formal expansion of $f(x)$,

$$
f(x) \sim \sum_{k=0}^{\infty} a_{k} p_{k}(x),
$$

then we can write a formal expansion for $I(f ; \lambda)$ in terms of the $q_{n}(\lambda)$,

$$
I(f ; \lambda) \sim \sum_{k=0}^{\infty} a_{k} q_{k}(\lambda) .
$$

Hence, an approximation to $I(f ; \lambda)$ will be given by the truncated sum

$$
S_{N}(f ; \lambda):=\sum_{k=0}^{N} a_{k} q_{k}(\lambda) .
$$

If we now have a sequence of integration rules

$$
Q_{m}(g):=\sum_{i=1}^{m} w_{i m} g\left(x_{i m}\right)
$$

which converges to

$$
I(g):=\int_{-1}^{1} w(x) g(x) d x
$$

for all $g \in C[-1,1]$ or all $g \in R[-1,1]$, the space of bounded Riemann integrable functions on $[-1,1]$, and if we approximate the Fourier coefficients $a_{k}$ by

$$
a_{k m}:=Q_{m}\left(f p_{k}\right)
$$


then, in general, we obtain a noninterpolatory integration rule for $I(f ; \lambda)$, namely

$$
Q_{m}^{N}(f ; \lambda):=\sum_{k=0}^{N} a_{k m} q_{k}(\lambda)
$$

The approximations $Q_{m}^{N}(f ; \lambda)$ can be evaluated in a stable manner using backward recursion by the algorithm given in [15], provided that we have the value of $q_{0}(\lambda)$. We can also express $Q_{m}^{N}(f ; \lambda)$ in a Lagrangian form that is more useful in the numerical solution of integral equations:

$$
Q_{m}^{N}(f ; \lambda)=\sum_{i=1}^{m} w_{i m}^{N}(\lambda) f\left(x_{i m}\right)
$$

where the weights

$$
w_{i m}^{N}(\lambda):=w_{i m} \sum_{k=0}^{N} p_{k}\left(x_{i m}\right) q_{k}(\lambda), \quad i=1,2, \ldots, m,
$$

can also be evaluated in a stable manner by the backward recursion algorithm [15].

As indicated above, this general approach to the numerical evaluation of CPV integrals appears in Henrici [8, pp. 139-142]. However, there is no discussion there of convergence or of the integration rules $Q_{m}(g)$. In fact, it is precisely the freedom in the choice of these rules, subject only to the condition that they converge to $I(g)$ for all $g \in C[-1,1]$ or all $g \in R[-1,1]$, that affords this method for evaluating CPV integrals considerable interest. Thus, if $f$ is well behaved in most of the interval $[-1,1]$, but is irregular over a small subinterval $[a, b] \subset[-1,1]$, then we can concentrate most of our integration points $x_{i m}$ in $[a, b]$.

This was also done by Gerasoulis [7] using a different approach, and the results he achieved were a considerable improvement over those achieved using a conventional spacing of integration points. There have been many approaches to noninterpolatory integration of CPV integrals [4], [14], [17], but these two are the only ones that cater to the situation indicated above.

In Section 2, we state and prove Theorems 1 to 5 , which deal with convergence of $S_{N}(f ; \lambda)$ to $I(f ; \lambda)$. In Section 3 , we state and prove Theorems 6 to 8 , which deal with the convergence of $Q_{m}^{N}(f ; \lambda)$ to $I(f ; \lambda)$ as $m$ and $N \rightarrow \infty$. It turns out that in the general case we shall be able to prove convergence only for the iterated limit

$$
\lim _{N \rightarrow \infty} \lim _{m \rightarrow \infty} Q_{m}^{N}(f ; \lambda) .
$$

In fact, we shall show that we cannot in general expect convergence of the double limit. However, in certain cases where we can convert the double limit to a single limit in which $m$ depends on $N$ in some specific manner, we shall again be able to prove convergence. A similar approach was used by Dagnino [3] in studying the convergence of noninterpolatory product integration rules.

2. Convergence Results for $S_{N}(f ; \lambda)$. Before we can study the convergence of $Q_{m}^{N}(f ; \lambda)$ to $I(f ; \lambda)$, we must establish the convergence of $S_{N}(f ; \lambda)$ to $I(f ; \lambda)$. To this end, we shall use the methods presented in Natanson [11] and Freud [5] for 
proving convergence of orthonormal expansions. Since the proofs in [11] depend on the Christoffel-Darboux formula

$$
\sum_{k=0}^{N} p_{k}(x) p_{k}(y)=\alpha_{N+1} \frac{p_{N+1}(x) p_{N}(y)-p_{N}(x) p_{N+1}(y)}{x-y}
$$

we shall first establish an analogous formula for the sum

$$
K_{N}(x, \lambda):=\sum_{k=0}^{N} p_{k}(x) q_{k}(\lambda)
$$

Throughout, $C, C_{1}, C_{2}, \ldots$, and $B, B_{1}, B_{2}, \ldots$ denote positive constants independent of $N, m, x$ and $\lambda$.

LEMMA 1. Let $\left\{p_{n}\right\}_{0}^{\infty}$ be a sequence of orthonormal polynomials on $[-1,1]$, with respect to $w \in \mathscr{A}$, and let $q_{n}(\lambda):=I\left(p_{n} ; \lambda\right), n=1,2,3, \ldots$, exist for a given $\lambda \in(-1,1)$. Then, for $N=1,2,3, \ldots$,

$$
K_{N}(x, \lambda)=\frac{\alpha_{N+1}}{\left\{p_{N+1}(x) q_{N}(\lambda)-p_{N}(x) q_{N+1}(\lambda)\right\}+1}-
$$

Proof. We have from (5) and (7) that for $k=0,1,2, \ldots$,

$$
x p_{k}(x)=\alpha_{k+1} p_{k+1}(x)+\beta_{k+1} p_{k}(x)+\alpha_{k} p_{k-1}(x),
$$

and

$$
\lambda q_{k}(\lambda)=\alpha_{k+1} q_{k+1}(\lambda)+\beta_{k+1} q_{k}(\lambda)+\alpha_{k} q_{k-1}(\lambda) .
$$

Multiply (22) by $q_{k}(\lambda)$ and multiply (23) by $p_{k}(x)$; then subtract the two and sum from $k=0$ to $N$. This yields

$$
\begin{aligned}
(x-\lambda) K_{N}(x, \lambda)= & \alpha_{N+1}\left\{p_{N+1}(x) q_{N}(\lambda)-p_{N}(x) q_{N+1}(\lambda)\right\} \\
& -\alpha_{0}\left\{p_{0}(x) q_{-1}(\lambda)-p_{-1}(x) q_{0}(\lambda)\right\} .
\end{aligned}
$$

Since $p_{-1}(x) \equiv 0, q_{-1}(\lambda) \equiv-1$ and $\alpha_{0}=m_{0}^{1 / 2}=1 / p_{0},(21)$ follows.

COROLlaRY 1. The sum $K_{N}(x, \lambda)$ can also be written as

$$
\begin{aligned}
K_{N}(x, \lambda) & \\
= & \alpha_{N+1}\left\{\frac{p_{N+1}(x)\left(q_{N}(\lambda)-q_{N}(x)\right)-p_{N}(x)\left(q_{N+1}(\lambda)-q_{N+1}(x)\right)}{x-\lambda}\right\} \\
& =\alpha_{N+1}\left\{\frac{q_{N+1}(\lambda)\left(p_{N}(\lambda)-p_{N}(x)\right)-q_{N}(\lambda)\left(p_{N+1}(\lambda)-p_{N+1}(x)\right)}{x-\lambda}\right\} .
\end{aligned}
$$

Proof. If we set $x=\lambda$ in (24), we find that

$$
\begin{aligned}
& \alpha_{N+1}\left\{p_{N+1}(x) q_{N}(x)-p_{N}(x) q_{N+1}(x)\right\} \\
& \quad=-1=\alpha_{N+1}\left\{p_{N+1}(\lambda) q_{N}(\lambda)-p_{N}(\lambda) q_{N+1}(\lambda)\right\} .
\end{aligned}
$$

Substituting into (21) yields (25).

Before proving some convergence theorems for $S_{N}(f ; \lambda)$, we recall some definitions and results connected with the existence of $I(f ; \lambda)[1]$. We say that a function $f$ is of Dini type on an interval $I$ of length $l(I)$, and write $f \in D T(I)$, if

$$
\int_{0}^{l(I)} \omega_{I}(f ; t) t^{-1} d t<\infty
$$


where $\omega_{I}(f ; t)$ is the ordinary modulus of continuity of $f$ on $I$, defined by

$$
\omega_{I}(f ; t):=\sup _{\substack{|x-y| \leq t \\ x, y \in I}}|f(x)-f(y)|, \quad t>0 .
$$

Obviously, if $f \in D T(I)$, then $f \in C(I)$. Furthermore, it can easily be shown that if $f \in D T(I)$, then $f$ satisfies the Dini-Lipschitz condition on $I$, that is

$$
\lim _{t \rightarrow 0+} \omega_{I}(f ; t) \log t=0 .
$$

Finally, it is well known that if $\lambda \in(-1,1)$ and if for some small enough $\varepsilon>0$, $f \in D T(\lambda-\varepsilon, \lambda+\varepsilon) \cap R[-1,1]$ and $w \in D T(\lambda-\varepsilon, \lambda+\varepsilon) \cap \mathscr{A}$, then $I(f ; \lambda)$ exists. Hence, to ensure the existence of $I(f ; \lambda)$ for all $\lambda \in(-1,1)$, it is sufficient to require that $f \in R[-1,1]$ and $w \in \mathscr{A}$ belong to $D T(-1,1)$.

We are now ready to prove some convergence results about $S_{N}(f ; \lambda)$ corresponding to the convergence theorems for orthonormal expansions in [11]. As usual, for $w \in \mathscr{A}$ and $0<p<\infty$, we let

$$
L_{p, w}:=\left\{g:[-1,1] \rightarrow \mathbf{R} \mid g \text { is measurable and } \int_{-1}^{1} w(x)|g(x)|^{p} d x<\infty\right\} .
$$

THEOREM 1. Assume that for some $\lambda \in(-1,1), I(f ; \lambda)$ exists, that

$$
\sup _{k}\left|q_{k}(\lambda)\right| \leq B<\infty
$$

and that

$$
\varphi_{\lambda}(x):=(f(x)-f(\lambda)) /(x-\lambda), \quad x \in[-1,1],
$$

belongs to $L_{2, w}$. Then

$$
\lim _{N \rightarrow \infty} S_{N}(f ; \lambda)=I(f ; \lambda) .
$$

Proof. Multiply (21) by $w(x)(f(x)-f(\lambda))$ and integrate between -1 and 1 . We obtain

$$
\begin{aligned}
& S_{N}(f ; \lambda)-f(\lambda) q_{0}(\lambda) / p_{0} \\
& \quad=\alpha_{N+1}\left\{c_{N+1} q_{N}(\lambda)-c_{N} q_{N+1}(\lambda)\right\}+I(f ; \lambda)-f(\lambda) q_{0}(\lambda) / p_{0},
\end{aligned}
$$

where $c_{k}:=\left(\varphi_{\lambda}, p_{k}\right)$ is the $k$ th Fourier coefficient of $\varphi_{\lambda}$ with respect to $p_{k}$. Since $\varphi_{\lambda} \in L_{2, w}, c_{k} \rightarrow 0$ as $k \rightarrow \infty$. Hence, since $\alpha_{N+1} \leq 1$ [5, p. 41], while (30) holds, we obtain (32).

An important special case of this theorem is that of the generalized smooth Jacobi weight (we write $w \in$ GSJ), studied by Nevai [13, p. 673], among others. It is defined by

$$
w(x):=\psi(x) \prod_{j=0}^{m+1}\left|x-t_{j}\right|^{\gamma_{j}}, \quad x \in[-1,1],
$$

where $m \geq 0,-1=t_{0}<t_{1}<\cdots<t_{m}<t_{m+1}=1, \gamma_{j}>-1, j=0,1,2, \ldots, m+1$, $\psi \in D T(-1,1)$ and $\psi(x)>0$ in $[-1,1]$. Clearly, if

$$
\mathscr{D}:=[-1,1] \backslash\left\{t_{0}, t_{1}, \ldots, t_{m+1}\right\},
$$

then $w \in \mathscr{A} \cap D T(\mathscr{D})$, so that if $\lambda \in \mathscr{D}$ and $f \in D T(\lambda-\varepsilon, \lambda+\varepsilon) \cap R[-1,1]$ for some $\varepsilon>0$, then $I(f ; \lambda)$ exists. Furthermore, Criscuolo and Mastroianni [2] have shown that if $w \in$ GSJ, then (30) holds for $\lambda \in \mathscr{D}$, and uniformly in any closed 
subset of $\mathscr{D}$. Hence, we have the following corollary:

COROLlaRY 2. If $w \in$ GSJ and $f \in D T(\lambda-\varepsilon, \lambda+\varepsilon) \cap R[-1,1]$ for some $\lambda \in \mathscr{D}$ and some small enough $\varepsilon>0$, while $\varphi_{\lambda} \in L_{2, w}$, then (32) holds.

In the sequel, we use the norm $\|f\|:=\max _{[-1,1]}|f(x)|$ for any $f \in C[-1,1]$.

THEOREM 2. If (30) holds for some $\lambda \in(-1,1)$, if

$$
\sup _{k}\left\|p_{k}(x)\right\|<\infty
$$

and if $\varphi_{\lambda} \in L_{1, w}$, then (32) holds.

Proof. By Theorem 3 in $\left[11\right.$, p. 69], the Fourier coefficients $c_{k}$ of $\varphi_{\lambda}$ (defined by (31)) converge to 0 as $k \rightarrow \infty$ under the hypotheses of the theorem. Furthermore, since $\varphi_{\lambda} \in L_{1, w}, I(f ; \lambda)$ exists, as shown by the identity

$$
I(f ; \lambda)=\int_{-1}^{1} w(x) \varphi_{\lambda}(x) d x+f(\lambda) q_{0}(\lambda) / p_{0} .
$$

Hence (32) follows from (33).

COROllary 3. Assume that $w \in$ GSJ, where $\gamma_{0}, \gamma_{m+1} \leq-1 / 2$ and $\gamma_{j} \leq 0$, $j=1,2, \ldots, m$. Further assume that $\lambda \in \mathscr{D}$, and that $\varphi_{\lambda} \in L_{1, w}$. Then (32) holds.

Proof. By Nevai $[13$, p. 674, (16)], there exists $C>0$ such that for $x \in[-1,1]$ and $k=1,2,3, \ldots$,

$$
\left|p_{k}(x)\right| \leq C\left\{\left[w(x)\left(1-x^{2}\right)^{1 / 2}\right]^{-1 / 2}+1\right\} .
$$

Hence, under the hypotheses of the corollary, (36) is true. Furthermore, as above, (30) is true for all $\lambda \in \mathscr{D}$. Hence, by Theorem 2, (32) holds.

Theorems 1 and 2 are of a local nature, since they depend on the behavior of the Fourier coefficients $c_{k}$ of $\varphi_{\lambda}(x)$. The following is a global theorem, and its proof requires much more delicate analysis. The proof is modelled on the proof of Theorem 2 in $[11$, p. 95].

THEOREM 3. If $f \in D T[-1,1]$ and $w \in G S J$, then (32) holds uniformly for $\lambda$ in each compact subset of $\mathscr{D}$.

Proof. We first remark that $I(f ; \lambda)$ exists for all $\lambda \in \mathscr{D}$ and that $f$ satisfies the Dini-Lipschitz condition $(28)$ on $J:=[-1,1]$. We shall start by proving that

$$
L_{N}(\lambda):=\int_{-1}^{1} w(x)\left|K_{N}(x, \lambda)\right| d x
$$

is $O(\log N)$, uniformly in a given compact subset $\mathscr{K}$ of $\mathscr{D}$. We first establish this bound for the case $m=0$ in (34), that is when $w(x)$ has no zeros or infinities in $(-1,1)$. To this end, we write $L_{N}(\lambda)$ as the sum of five integrals

$$
\begin{aligned}
L_{N}(\lambda) & =\int_{-1}^{-1+h / 2}+\int_{-1+h / 2}^{\lambda-1 / N}+\int_{\lambda-1 / N}^{\lambda+1 / N}+\int_{\lambda+1 / N}^{1-h / 2}+\int_{1-h / 2}^{1} \\
& =: I_{1}+I_{2}+I_{3}+I_{4}+I_{5}
\end{aligned}
$$


and choose $N$ sufficiently large so that $[\lambda-1 / N, \lambda+1 / N] \subset \mathscr{D}$ for all $\lambda \in \mathscr{K}$ and choose $h>0$ so small that $\mathscr{K} \subset[-1+h, 1-h]$. We consider first $I_{1}$ and use (21) for $K_{N}(x, \lambda)$. Now, for $x \in[-1,-1+h / 2]$ and $\lambda \in \mathscr{K},|x-\lambda| \geq h / 2$. Further, since (30) holds uniformly for $\lambda \in \mathscr{K}$, since $\alpha_{N+1} \leq 1$, and since

$$
\begin{aligned}
\int_{-1}^{1} w(x)\left|p_{k}(x)\right| d x & \leq\left\{\int_{-1}^{1} w(x) d x\right\}^{1 / 2}\left\{\int_{-1}^{1} w(x) p_{k}^{2}(x) d x\right\}^{1 / 2} \\
& =\left\{\int_{-1}^{1} w(x) d x\right\}^{1 / 2},
\end{aligned}
$$

it follows that $I_{1}=O(1)$. Similarly, $I_{5}=O(1)$. For $x \in[\lambda+1 / N, 1-h / 2]$, it follows from (38) and the fact that (30) holds uniformly for $\lambda \in \mathscr{K}$, that

$$
\left|K_{N}(x, \lambda)\right| \leq C /|x-\lambda|
$$

where $C$ is independent of $N, x$ and $\lambda$. Hence,

$$
\begin{aligned}
I_{4} & \leq C^{-1} \int_{\lambda+1 / N}^{1} \frac{w(x)}{x-\lambda} d x \\
& \leq \int_{-1}^{1}\left|\frac{w(x)-w(\lambda)}{x-\lambda}\right| d x+w(\lambda) \int_{\lambda+1 / N}^{1} \frac{d x}{x-\lambda}=O(\log N) .
\end{aligned}
$$

Similarly, $I_{2}=O(\log N)$. Finally, since

$$
\left|K_{N}(x, \lambda)\right| \leq(N+1) \sup _{k}\left|p_{k}(x)\right| \sup _{k}\left|q_{k}(\lambda)\right|
$$

we obtain $I_{3}=O(1)$. Combining these estimates, we obtain

$$
\sup _{\lambda \in \mathscr{K}}\left|L_{N}(\lambda)\right| \leq C_{1} \log N
$$

for some $C_{1}$ independent of $N$. For the general case, we let $h$ be the distance of $\mathscr{K}$ from the set $T:=\left\{t_{0}, t_{1}, \ldots, t_{m+1}\right\}$ and denote by $U$ the subset of $[-1,1]$ such that the distance of $T$ to $U$ is at most $h / 2$. As before, we can show that

$$
\int_{U} w(x)\left|K_{N}(x, \lambda)\right| d x=O(1)
$$

and that

$$
\int_{V_{N}} w(x)\left|K_{N}(x, \lambda)\right| d x=O(\log N),
$$

where $V_{N}:=[-1,1] \backslash([\lambda-1 / N, \lambda+1 / N] \cup U)$. If we choose $N$ large enough so that $1 / N<h$, we obtain $(40)$.

Next, let $P_{N}^{*}$ be the polynomial of best approximation to $f$ in the uniform norm, let $r_{N}:=f-P_{N}^{*}$, and let $E_{N}(f):=\left\|r_{N}\right\|$. Since $f$ satisfies (28) on $J$, it follows from Jackson's Theorems that

$$
\lim _{N \rightarrow \infty} E_{N}(f) \log N=0 .
$$

Now, for any $g \in C[-1,1]$, we have

$$
\left|S_{N}(g ; \lambda)\right|=\left|\left(g, K_{N}(x, \lambda)\right)\right| \leq\|g\| L_{N}(\lambda) .
$$


Hence, uniformly for $\lambda \in \mathscr{K}$, we have from (40) and (41),

$$
\lim _{N \rightarrow \infty}\left|S_{N}\left(r_{N} ; \lambda\right)\right|=0 \text {. }
$$

Since

$$
I\left(P_{N}^{*} ; \lambda\right)=S_{N}\left(P_{N}^{*} ; \lambda\right)
$$

we have

$$
I(f ; \lambda)-S_{N}(f ; \lambda)=I\left(r_{N} ; \lambda\right)-S_{N}\left(r_{N} ; \lambda\right),
$$

and it thus remains to show that

$$
\lim _{N \rightarrow \infty}\left|I\left(r_{N} ; \lambda\right)\right|=0 .
$$

Now

$$
\begin{aligned}
I\left(r_{N} ; \lambda\right) & =\int_{-1}^{1} w(x) \frac{r_{N}(x)-r_{N}(\lambda)}{x-\lambda} d x+r_{N}(\lambda) q_{0}(\lambda) / p_{0} \\
& =\int_{-1}^{1} w(x) \frac{r_{N}(x)-r_{N}(\lambda)}{x-\lambda} d x+o(1) .
\end{aligned}
$$

Furthermore, as in [1],

$$
\begin{aligned}
\int_{-1}^{1} w(x) \frac{r_{N}(x)-r_{N}(\lambda)}{x-\lambda} d x & =\int_{-1}^{\lambda-1 / N}+\int_{\lambda-1 / N}^{\lambda+1 / N}+\int_{\lambda+1 / N}^{1} \\
& =: J_{1}+J_{2}+J_{3} .
\end{aligned}
$$

Here

$$
\left|J_{1}\right| \leq 2 E_{N}(f) \int_{-1}^{\lambda-1 / N} \frac{w(x)}{|x-\lambda|} d x=E_{N}(f) O(\log N)=o(1) \quad \text { as } N \rightarrow \infty .
$$

Similarly, $J_{3}=o(1)$ as $N \rightarrow \infty$. Finally,

$$
\begin{array}{rl}
\int_{\lambda-1 / N}^{\lambda+1 / N} & w(x) \frac{r_{N}(x)-r_{N}(\lambda)}{x-\lambda} d x \\
& =\int_{\lambda-1 / N}^{\lambda+1 / N} w(x) \frac{f(x)-f(\lambda)}{x-\lambda} d x-\int_{\lambda-1 / N}^{\lambda+1 / N} w(x) \frac{P_{N}^{*}(x)-P_{N}^{*}(\lambda)}{x-\lambda} d x .
\end{array}
$$

Since $f \in D T[-1,1]$, the first integral on the right-hand side is $o(1)$. As for the second integral, we have from [9] that

$$
\begin{aligned}
\left|\frac{P_{N}^{*}(x)-P_{N}^{*}(\lambda)}{x-\lambda}\right| & \leq \max \left\{\left|P_{N}^{* \prime}(t)\right|: t \in[\lambda-1 / N, \lambda+1 / N]\right\} \\
& \leq C N \omega(f ; 1 / N) .
\end{aligned}
$$

Hence

$$
\begin{array}{rl}
\int_{\lambda-1 / N}^{\lambda+1 / N} & w(x)\left|\frac{P_{N}^{*}(x)-P_{N}^{*}(\lambda)}{x-\lambda}\right| d x \\
& \leq 2 C \omega(f ; 1 / N) \max \{w(x): x \in[\lambda-1 / N, \lambda+1 / N]\} \\
& \rightarrow 0 \quad \text { as } N \rightarrow \infty
\end{array}
$$

since $w(x)$ is uniformly bounded above for $\lambda \in \mathscr{K}$ and $N$ large enough. This completes our proof.

Remark. Theorem 3 is similar to Theorem 2.2 in [1]. By following the proof of Theorem 3, we can prove a result similar to Theorem 2.1 in [1], namely, that if $f$ satisfies (28) on $J$, if $w \in \mathrm{GSJ}$, and if for some $\lambda \in \mathscr{D}, I(f ; \lambda)$ exists, then (32) 
holds. The proof of Theorem 3 holds in this case too, except that we must show that

$$
J_{N}:=\int_{\lambda-1 / N}^{\lambda+1 / N} w(x) \frac{f(x)-f(\lambda)}{x-\lambda} d x=o(1), \quad N \rightarrow \infty .
$$

Since

$$
J_{0}:=\int_{-1}^{1} w(x) \frac{f(x)-f(\lambda)}{x-\lambda} d x=I(f ; \lambda)-f(\lambda) I(1 ; \lambda),
$$

and both $I(f ; \lambda)$ and $I(1 ; \lambda)$ exist, it follows that $J_{0}$ exists. Hence $J_{N}=o(1)$, and the proof is complete.

We now give some additional conditions for (32) to hold, which impose less restrictions on the weight function $w \in \mathscr{A}$, but require more smoothness of $f$. To this end, we first prove a lemma:

LEMMA 2. Let $w \in \mathscr{A}$, and assume that for some $\lambda \in(-1,1)$,

$$
\Gamma(\lambda):=\int_{-1}^{1}\left|\frac{w(x)-w(\lambda)}{x-\lambda}\right| d x<\infty,
$$

while for some positive $\varepsilon, B_{1}$ and $B_{2}$

$$
B_{1} \leq w(x) \leq B_{2} \quad \text { for }|x-\lambda| \leq 2 \varepsilon .
$$

Then there exists a constant $B_{3}>0$ such that

$$
T_{n-1}(\lambda):=\sum_{k=0}^{n-1} q_{k}^{2}(\lambda) \leq B_{3} n, \quad n=1,2,3, \ldots
$$

If $\Gamma(\lambda)$ is uniformly bounded and (43) holds uniformly for $\lambda \in[a-\varepsilon, b+\varepsilon] \subset[-1,1]$, then (44) holds uniformly for $\lambda \in[a, b]$.

Proof. We first establish the following analogue of the Christoffel function extremum problem, noting that in essence, it is contained in [6]: Defining

$$
\rho_{n}(w ; \lambda):=\inf \left\{\frac{I\left(P^{2}\right)}{(I(P ; \lambda))^{2}}: P \in \mathscr{P}_{n-1}, I(P ; \lambda) \neq 0\right\},
$$

where $\mathscr{P}_{m}$ denotes the set of all polynomials of degree $\leq m$, we have

$$
\rho_{n}(w ; \lambda)=1 / T_{n-1}(\lambda) .
$$

To see this, we note that for any $P \in \mathscr{P}_{n-1}$, we can write

$$
P(x)=\sum_{k=0}^{n-1} a_{k} p_{k}(x), \quad \text { where } a_{k}:=\left(P, p_{k}\right), k=0,1,2, \ldots n-1 .
$$

Hence

so that

$$
\begin{aligned}
|I(P ; \lambda)| & =\left|\sum_{k=0}^{n-1} a_{k} q_{k}(\lambda)\right| \leq\left\{\sum_{k=0}^{n-1} a_{k}^{2}\right\}^{1 / 2}\left\{T_{n-1}(\lambda)\right\}^{1 / 2} \\
& =\left\{I\left(P^{2}\right)\right\}^{1 / 2}\left\{T_{n-1}(\lambda)\right\}^{1 / 2}
\end{aligned}
$$

$$
\rho_{n}(w, \lambda) \geq 1 / T_{n-1}(\lambda) .
$$


On the other hand,

$$
\hat{P}(x):=\sum_{k=0}^{n-1} p_{k}(x) q_{k}(\lambda) \in \mathscr{P}_{n-1}
$$

and satisfies

$$
I(\hat{P} ; \lambda)=T_{n-1}(\lambda)=I\left(\hat{P}^{2}\right)
$$

Then (46) follows.

We now use (46) to prove (44). Choose $\varepsilon$ such that $[\lambda-2 \varepsilon, \lambda+2 \varepsilon] \subset[-1,1]$. Now for any $P \in \mathscr{P}_{n-1}$,

$$
\begin{aligned}
|I(P ; \lambda)| & =\mid \int_{|x-\lambda| \leq \varepsilon} P(x) \frac{w(x)-w(\lambda)}{x-\lambda} d x+w(\lambda) f_{|x-\lambda| \leq \varepsilon} \frac{P(x)}{x-\lambda} d x \\
& +\int_{|x-\lambda| \geq \varepsilon} \frac{w(x) P(x)}{x-\lambda} d x \mid \\
& \leq \Gamma(\lambda) \max _{|x-\lambda| \leq \varepsilon}|P(x)|+w(\lambda)\left|f_{|x-\lambda| \leq \varepsilon} \frac{P(x)}{x-\lambda} d x\right|+\varepsilon^{-1} I(|P|) .
\end{aligned}
$$

Next, let $\chi$ be the characteristic function of $[\lambda-\varepsilon, \lambda+\varepsilon]$, that is, $\chi(x):=1$ in $[\lambda-\varepsilon, \lambda+\varepsilon]$ and $\chi(x):=0$ elsewhere. We have from (45),

$$
\begin{aligned}
& \quad\left|f_{|x-\lambda| \leq \varepsilon} \frac{P(x)}{x-\lambda} d x\right|^{2} \leq \rho_{n}(\chi ; \lambda)^{-1} \int_{|x-\lambda| \leq \varepsilon} P^{2}(x) d x \\
& \quad \leq B_{1}^{-1} \rho_{n}(\chi ; \lambda)^{-1} \int_{|x-\lambda| \leq \varepsilon} P^{2}(x) w(x) d x \leq B_{1}^{-1} \rho_{n}(\chi ; \lambda)^{-1} I\left(P^{2}\right) .
\end{aligned}
$$

Furthermore, by standard estimates for Christoffel functions for the Legendre weight (cf. [12]),

$$
\max _{|x-\lambda| \leq \varepsilon}(P(x))^{2} \leq C n \int_{\lambda-2 \varepsilon}^{\lambda+2 \varepsilon} P^{2}(t) d t \leq C n B_{1}^{-1} I\left(P^{2}\right) .
$$

Combining (47), (48) and (49), and using the Cauchy-Schwarz inequality, we obtain

$$
|I(P ; \lambda)| \leq B_{4}\left\{n^{1 / 2} \Gamma(\lambda)+\rho_{n}^{-1 / 2}(\chi ; \lambda)+1\right\} I\left(P^{2}\right)^{1 / 2} .
$$

But

$$
\rho_{n}(\chi ; \lambda)^{-1}=\sum_{k=0}^{n-1} q_{k}^{2}(\chi ; \lambda) \leq B_{5} n
$$

since $q_{k}(\chi ; \lambda)$ is the function of the second kind associated with the Legendre weight shifted to $[\lambda-\varepsilon, \lambda+\varepsilon]$, so that $q_{k}(\chi ; \lambda)=O(1)$. Hence

$$
|I(P ; \lambda)| \leq B_{6} n^{1 / 2} I\left(P^{2}\right)^{1 / 2},
$$

so that

$$
1 / T_{n-1}(\lambda)=\rho_{n}(w ; \lambda) \geq B_{7} / n \text {. }
$$

If the assumptions on $\lambda$ hold uniformly in $[a-\varepsilon, b+\varepsilon]$, it is not difficult to modify the proof to hold uniformly in $[a, b]$.

We now prove the analogue of Theorem IV.1.2 in Freud [5, p. 139]. 
THEOREM 4. Let $w \in \mathscr{A}$ and assume that for some $\lambda \in(-1,1)$, (42) holds, while (43) holds for some positive $\varepsilon, B_{1}$ and $B_{2}$. Define for $n=1,2,3, \ldots$,

$$
E_{n}^{(2)}(f ; w):=\inf _{P \in \mathscr{P}_{n}}(f-P, f-P)^{1 / 2} .
$$

Then, if

$$
\sum_{n=1}^{\infty} E_{n}^{(2)}(f ; w) n^{-1 / 2}<\infty
$$

(32) holds. If $\Gamma(\lambda)$ is uniformly bounded for $\lambda \in[a-\varepsilon, b+\varepsilon] \subset[-1,1]$, while (43) holds uniformly for $\lambda \in[a-\varepsilon, b+\varepsilon]$, then (32) holds uniformly in $[a, b]$.

Proof. First recall the notation (8). For any positive integer $m$,

$$
\begin{aligned}
\sum_{k=2^{m}+1}^{2^{m+1}}\left|a_{k} q_{k}(\lambda)\right| & \leq\left\{\sum_{k=2^{m}+1}^{2^{m+1}} a_{k}^{2}\right\}^{1 / 2}\left\{\sum_{k=2^{m}+1}^{2^{m+1}} q_{k}^{2}(\lambda)\right\}^{1 / 2} \\
& \leq\left\{\sum_{k=2^{m}+1}^{\infty} a_{k}^{2}\right\}^{1 / 2}\left\{\sum_{k=0}^{2^{m+1}} q_{k}^{2}(\lambda)\right\}^{1 / 2} \\
& =E_{2^{m}}^{(2)}(f ; w)\left\{\sum_{k=0}^{2^{m+1}} q_{k}^{2}(\lambda)\right\}^{1 / 2} \\
& \leq C E_{2^{m}}^{(2)}(f ; w) 2^{m / 2}
\end{aligned}
$$

where the last inequality follows from (44). Since $E_{k}^{(2)}(f ; w)$ is nonincreasing with $k$,

$$
\begin{aligned}
2^{m / 2} E_{2^{m}}^{(2)}(f ; w) & \leq 2^{m / 2}\left\{2^{-m+1} \sum_{k=2^{m-1}+1}^{2^{m}} E_{k}^{(2)}(f ; w)\right\} \\
& =2^{1-m / 2} \sum_{k=2^{m-1}+1}^{2^{m}} E_{k}^{(2)}(f ; w) \leq 2 \sum_{k=2^{m-1}+1}^{2^{m}} E_{k}^{(2)}(f ; w) k^{-1 / 2} .
\end{aligned}
$$

Hence,

$$
\sum_{k=2}^{\infty}\left|a_{k} q_{k}(\lambda)\right|=\sum_{m=1}^{\infty} \sum_{k=2^{m-1}+1}^{2^{m}}\left|a_{k} q_{k}(\lambda)\right| \leq B \sum_{k=1}^{\infty} E_{k}^{(2)}(f ; w) k^{-1 / 2} .
$$

The next theorem is the analogue of Theorem IV.1.3 in Freud [5, p. 140].

THEOREM 5. Let $w$ and $\lambda$ be as in Theorem 4. Let $f \in C[-1,1]$ and for $J:=[-1,1]$, suppose that $w_{J}(f ; \delta)$ satisfies for some $\eta>0$,

$$
\lim _{\delta \rightarrow 0+} w_{J}(f ; \delta) \delta^{-1 / 2}|\log \delta|^{1+\eta}=0 .
$$

Then (32) holds. If $\Gamma(\lambda)$ is uniformly bounded for $\lambda \in[a-\varepsilon, b+\varepsilon] \subset[-1,1]$, while (43) holds uniformly for $\lambda \in[a-\varepsilon, b+\varepsilon]$, then (32) holds uniformly in $[a, b]$.

Proof. By Jackson's Theorem,

$$
E_{k}^{(2)}(f ; w) \leq B_{1} w_{J}\left(f ; k^{-1}\right) \leq B_{1} k^{-1 / 2}|\log k|^{-1-\eta} .
$$


3. Convergence Results for $Q_{m}^{N}(f ; \lambda)$. We are now ready to prove our convergence theorems for $Q_{m}^{N}(f ; \lambda)$. First a result on the iterated limit.

THEOREM 6. Assume that $f \in R[-1,1]$, that $I(f ; \lambda)$ exists and that $w \in \mathscr{A}$ and $\lambda \in[-1,1]$ are such that $(32)$ holds. Let $\left\{Q_{m}(\cdot)\right\}_{m=1}^{\infty}$ be a sequence of integration rules such that for all $g \in R[-1,1]$,

$$
\lim _{m \rightarrow \infty} Q_{m}(g)=I(g)
$$

Then

$$
\lim _{N \rightarrow \infty} \lim _{m \rightarrow \infty} Q_{m}^{N}(f ; \lambda)=I(f ; \lambda) .
$$

Proof. It suffices to show that for each fixed $N$,

$$
\lim _{m \rightarrow \infty} Q_{m}^{N}(f ; \lambda)=S_{N}(f ; \lambda)
$$

since

$$
I(f ; \lambda)=S_{N}(f ; \lambda)+\sum_{k=N+1}^{\infty} a_{k} q_{k}(\lambda)=S_{N}(f ; \lambda)+o(1) .
$$

For fixed $N$, we choose $m$ sufficiently large so that

$$
\left|a_{k m}-a_{k}\right| \leq \varepsilon \max _{0 \leq k \leq N}\left|q_{k}(\lambda)\right| /(N+1), \quad k=0,1,2, \ldots, N,
$$

yielding the theorem.

Even though we have convergence of the iterated limit (53), we cannot in general have convergence of the double limit (that is the limit with $m$ and $N \rightarrow \infty$ simultaneously), as illustrated by the following simple example:

Example 1. Let

$$
w(x):=\left(1-x^{2}\right)^{-1 / 2} \quad \text { and } \quad f(x) \equiv 1, \quad x \in(-1,1),
$$

and let $Q_{m}(\cdot)$ be the Gauss-Chebyshev rule

$$
Q_{m}(g):=\frac{\pi}{m} \sum_{i=1}^{m} g\left(\cos \frac{2 i-1}{2 m} \pi\right) .
$$

Then, with $N=2 m$, we have that

$$
Q_{m}^{2 m}(f ; \lambda)=\sum_{k=0}^{2 m} Q_{m}\left(f p_{k}\right) q_{k}(\lambda)=\sum_{k=0}^{2 m} Q_{m}\left(p_{k}\right) q_{k}(\lambda)
$$

Since $Q_{m}(g)$ is exact for all $g \in \mathscr{P}_{2 m-1}$,

$$
Q_{m}\left(p_{k}\right)=I\left(p_{k}\right)=\int_{-1}^{1} w(x) p_{k}(x) d x, \quad 0 \leq k \leq 2 m-1,
$$

so that

$$
Q_{m}\left(p_{0}\right)=p_{0} \pi \quad \text { and } \quad Q_{m}\left(p_{k}\right)=0, \quad k=1,2, \ldots, 2 m-1
$$


Furthermore,

$$
\begin{aligned}
Q_{m}\left(p_{2 m}\right) & =\frac{\pi}{m} \sum_{i=1}^{m}\left(\frac{2}{\pi}\right)^{1 / 2} T_{2 m}\left(\cos \frac{2 i-1}{2 m} \pi\right) \\
& =(2 \pi)^{1 / 2} m^{-1} \sum_{i=1}^{m} \cos (2 i-1) \pi=-(2 \pi)^{1 / 2}
\end{aligned}
$$

Hence

$$
Q_{m}^{2 m}(f ; \lambda)=\pi^{1 / 2} q_{0}(\lambda)-(2 \pi)^{1 / 2} q_{2 m}(\lambda)
$$

But (see, for example, [8, p. 148])

$$
q_{0}(\lambda)=I(f ; \lambda)=0
$$

so

$$
Q_{m}^{2 m}(f ; \lambda)-I(f ; \lambda)=-(2 \pi)^{1 / 2} q_{2 m}(\lambda),
$$

which does not go to zero for any nonzero $\lambda \in(-1,1)$ as $m \rightarrow \infty$, inasmuch as $q_{2 m}(\lambda)=(2 / \pi)^{1 / 2} U_{2 m+1}(\lambda)$, where $U_{2 m+1}(\lambda)$ is the Chebyshev polynomial of the second kind of degree $2 m+1$.

Example 1 shows that at least in general, converting the iterated limit to a single limit does not lead to convergence. However, there are cases where this procedure will work. One simple example occurs when $m=N+1$ and $Q_{m}(\cdot)$ is the Gauss integration rule with respect to $w$. In this case, it turns out that

$$
Q_{N+1}^{N}(f ; \lambda)=I\left(L_{N+1} ; \lambda\right),
$$

where $L_{N+1}$ is the Lagrange interpolation polynomial of degree $\leq N$ interpolating $f$ at the zeros of $p_{N+1}$. This follows since

$$
\begin{aligned}
I\left(L_{N+1} ; \lambda\right) & =\sum_{k=0}^{N}\left(L_{N+1}, p_{k}\right) q_{k}(\lambda)=\sum_{k=0}^{N} Q_{N+1}\left(L_{N+1} p_{k}\right) q_{k}(\lambda) \\
& =\sum_{k=0}^{N} Q_{N+1}\left(f p_{k}\right) q_{k}(\lambda)=Q_{N+1}^{N}(f ; \lambda)
\end{aligned}
$$

(see, for example, [16, pp. 1250-1251]). Since it has been shown in [1] that for $w \in \mathrm{GSJ}$,

$$
\lim _{N \rightarrow \infty} I\left(L_{N+1}, \lambda\right)=I(f ; \lambda)
$$

we have that for the sequence of Gauss rules $\left\{Q_{m}(\cdot)\right\}_{m=1}^{\infty}$ associated with $w \in$ GSJ,

$$
\lim _{N \rightarrow \infty} Q_{N+1}^{N}(f ; \lambda)=I(f ; \lambda) \text {. }
$$

We can generalize this result to any sequence of integration rules $\left\{Q_{m}(\cdot)\right\}_{m=1}^{\infty}$ that is ultimately exact for all polynomials, that is $Q_{m}(g)=I(g)$ for all $g \in \mathscr{P}_{n}$ and all $m \geq m(n)$. A particular instance of this, that allows points to be concentrated in regions where the behaviour of $f$ is problematic, is rules exact for piecewise polynomials of increasing degree.

In the general situation, if the weights $w_{i m}$ and the points $x_{i m}$ in a sequence of rules $\left\{Q_{m}(\cdot)\right\}_{m=1}^{\infty}$ are such that

$$
\sum_{i=1}^{m}\left|w_{i m}^{N}(\lambda)\right|=O(\log N)
$$


if $f \in D T[-1,1]$ and if $w \in G S J$, then we have that

$$
\lim _{N \rightarrow \infty} Q_{m(2 N)}^{N}(f ; \lambda)=I(f ; \lambda) .
$$

Here $m(2 N)$ denotes the least integer $m$ such that $Q_{m}(g)=I(g)$ for all $g \in \mathscr{P}_{2 N}$. The proof follows standard lines, namely

$$
I(f ; \lambda)=I\left(P_{N}^{*} ; \lambda\right)+I\left(r_{N} ; \lambda\right),
$$

where, as above, $P_{N}^{*} \in \mathscr{P}_{N}$ is the polynomial of best approximation to $f$ in the uniform norm and $r_{N}:=f-P_{N}^{*}$. Since, by hypothesis, $Q_{m}\left(g p_{k}\right)=I\left(g p_{k}\right)$ for all $k \leq N$, all $m \geq m(2 N)$ and all $g \in \mathscr{P}_{N}$, it follows that

$$
Q_{m}^{N}(f ; \lambda)=I\left(P_{N}^{*} ; \lambda\right)+Q_{m}^{N}\left(r_{N} ; \lambda\right) \text {. }
$$

Hence

$$
\begin{aligned}
\mid I(f ; \lambda)-Q_{m}^{N}(f ; \lambda) & \leq\left|I\left(r_{N} ; \lambda\right)\right|+\left|Q_{m}^{N}\left(r_{N} ; \lambda\right)\right| \\
& \leq\left|I\left(r_{N} ; \lambda\right)\right|+\sum_{i=1}^{m}\left|w_{i m}^{N}(\lambda)\right|\left\|r_{N}\right\| .
\end{aligned}
$$

As in the proof of Theorem 3 above, $I\left(r_{N} ; \lambda\right)=o(1)$, and since $f$ satisfies (28), the second term is also $o(1)$ from (56), proving (57).

What about conditions on $w_{i m}$ and $x_{i m}$ that ensure (56)? We shall prove

LEMMA 3. With the above notation, if

$$
h_{i m}:=x_{i+1, m}-x_{i m} \leq C_{1} / N
$$

for some $C_{1}>0$, uniformly for all $i$ and $m \geq m(2 N)$, while

$$
\left|w_{i m}\right| / w\left(x_{i m}\right) \leq C_{2}\left(h_{i-1, m}+h_{i m}\right),
$$

then (56) holds whenever $w \in$ GSJ and $\lambda \in \mathscr{D}$.

Proof. As in the proof of Theorem 3, we consider first the case $m=0$ in (34), and we decompose the sum on the left-hand side of (56) into five sums:

$$
\begin{aligned}
\sum_{i=1}^{m}\left|w_{i m}^{N}(\lambda)\right|= & \sum_{x_{i m} \leq-1+\delta}+\sum_{\substack{\lambda-x_{i m}>2 C_{1} / N \\
x_{i m}>-1+\delta}}+\sum_{\left|x_{i m}-\lambda\right| \leq 2 C_{1} / N} \\
& +\sum_{\substack{x_{i m}-\lambda>2 C_{1} / N \\
x_{i m}<1-\delta}}+\sum_{x_{i m} \geq 1-\delta} \\
= & : \sum_{1}+\sum_{2}+\sum_{3}+\sum_{4}+\sum_{5},
\end{aligned}
$$

where $\delta$ is some sufficiently small positive number. Now by (17) and (21), and the uniform boundedness of $\left\{q_{k}(\lambda)\right\}_{0}^{\infty}$,

$$
\begin{aligned}
\sum_{1} & \leq(1-\delta+\lambda)^{-1} O(1) \sum w_{i m}\left\{\left|p_{N}\left(x_{i m}\right)\right|+\left|p_{N+1}\left(x_{i m}\right)\right|\right\} \\
& \leq O(1) \int_{-1}^{-1+\delta+C_{1} / N} w(x)\left\{\left|p_{N}(x)\right|+\left|p_{N+1}(x)\right|\right\} d x
\end{aligned}
$$

(by Theorem 5 in $[10$, p. 534])

$$
\leq O(1)\left\{\int_{-1}^{1} w(x) d x\right\}^{1 / 2}=O(1)
$$


Similarly, $\sum_{5}=O(1)$. Next,

$$
\begin{aligned}
\sum_{2} & =O(1) \sum\left|w_{i m}\right| /\left(\lambda-x_{i m}\right) \\
& \leq O(1) \int_{-1}^{\lambda-C_{1} / N} d x /(\lambda-x)=O(\log N)
\end{aligned}
$$

by uniform boundedness of $p_{N}, q_{N}$ and $w$. Similarly, $\sum_{4}=O(\log N)$. Finally,

$$
\begin{aligned}
\sum_{3} & =O(1) \sum_{\left|x_{i m}-\lambda\right| \leq 2 C_{1} / N}\left|w_{i m}\right| \sum_{k=0}^{N}\left|p_{k}\left(x_{i m}\right) q_{N}(\lambda)\right| \\
& =O(1)(N+1) \sum_{\left|x_{i m}-\lambda\right| \leq 2 C_{1} / N}\left|w_{i m}\right| \\
& =O(1), \quad
\end{aligned}
$$

which proves the lemma for the case $m=0$. For the general case, we enclose each of the interior singularities of $w$ in a small interval avoiding $\lambda$ and treat the $w_{i m}$ associated with these intervals in the same manner as $\sum_{1}$.

The assertion (57) is a special case of the following theorem:

THEOREM 7. Suppose that for $m=1,2,3, \ldots$, the rule $Q_{m}(\cdot)$ has precision $\pi_{m}>N_{m}$, that $t_{m}:=\min \left\{N_{m}, \pi_{m}-N_{m}\right\}$ satisfies

$$
\lim _{m \rightarrow \infty} t_{m}=\infty
$$

and that

$$
\sum_{i=1}^{m}\left|w_{i m}^{N_{m}}(\lambda)\right| \leq C \log t_{m}, \quad m=1,2,3, \ldots
$$

Assume that $f \in C[-1,1]$ satisfies (28) with $I=[-1,1]$, that $I(f ; \lambda)$ exists, that $q_{0}(\lambda)$ is finite and that $w(x)$ is bounded above in a neighborhood of $\lambda$. Then

$$
\lim _{m \rightarrow \infty} Q_{m}^{N_{m}}(f ; \lambda)=I(f ; \lambda) .
$$

Proof. If $P \in \mathscr{P}_{t_{m}}$, then

$$
\begin{aligned}
Q_{m}^{N_{m}}(P ; \lambda) & =\sum_{k=0}^{N_{m}} Q_{m}\left(P p_{k}\right) q_{k}(\lambda)=\sum_{k=0}^{N_{m}}\left(P, p_{k}\right) q_{k}(\lambda) \\
& =\sum_{k=0}^{t_{m}}\left(P, p_{k}\right) q_{k}(\lambda)=I(P ; \lambda)
\end{aligned}
$$

since $t_{m} \leq N_{m}$. Then, if $P_{m}^{*} \in \mathscr{P}_{t_{m}}$ is the polynomial of best approximation to $f$ in the uniform norm, and if $r_{m}:=f-P_{m}^{*}$, then as above, for $m$ sufficiently large 
so that $\left[\lambda-1 / t_{m}, \lambda+1 / t_{m}\right] \subset[-1,1]$,

$$
\begin{aligned}
\left|Q_{m}^{N_{m}}(f ; \lambda)-I(f ; \lambda)\right|=\left|Q_{m}^{N_{m}}\left(r_{m} ; \lambda\right)-I\left(r_{m} ; \lambda\right)\right| & w(x) \frac{\left|r_{m}(x)\right|}{|x-\lambda|} d x \\
\leq & \sum_{i=1}^{m}\left|w_{i m}^{N_{m}}(\lambda)\right|\left\|r_{m}\right\|+\int_{|\lambda-x| \geq 1 / t_{m}} \frac{w(x) r_{m}(x)}{x-\lambda} d x \mid \\
& +\left|f_{|\lambda-x| \leq 1 / t_{m}} w\right| \\
\leq & C \log t_{m} \omega\left(f ; t_{m}^{-1}\right)+C_{1}\left\|r_{m}\right\| \log t_{m} \\
& +\left|\int_{|\lambda-x| \leq 1 / t_{m}} w(x) \frac{f(x)-f(\lambda)}{x-\lambda} d x\right| \\
& +\left|\int_{|\lambda-x| \leq 1 / t_{m}} w(x) \frac{P_{m}^{*}(x)-P_{m}^{*}(\lambda)}{x-\lambda} d x\right| \\
& +\left|r_{m}(\lambda)\right|\left|f_{|\lambda-x| \leq 1 / t_{m}} \frac{w(x)}{x-\lambda} d x\right| \\
\leq & o(1)+o(1)+o(1)+o(1)+o(1),
\end{aligned}
$$

by the arguments used in the proof of Theorem 3 and the fact that $w$ is bounded above near $\lambda$.

We conclude with an almost trivial theorem that gives necessary and sufficient conditions for the convergence of a sequence of approximations $\left\{Q_{m}^{N_{m}}(f ; \lambda)\right\}_{m=1}^{\infty}$. It shows that we must choose $N_{m}$ in such a way that $Q_{m}\left(f p_{k}\right)$ is small for all $k$ large enough with $k \leq N_{m}$ :

THEOREM 8. Assume that for all $g \in R[-1,1]$,

$$
\lim _{m \rightarrow \infty} Q_{m}(g)=I(g)
$$

that $I(f ; \lambda)$ exists and that $(32)$ holds. Then, given a sequence $\left\{\left(m, N_{m}\right\}\right\}_{m=1}^{\infty}$ of pairs of positive integers with

we have that

$$
\lim _{m \rightarrow \infty} N_{m}=\infty
$$

$$
\lim _{m \rightarrow \infty} Q_{m}^{N_{m}}(f ; \lambda)=I(f ; \lambda)
$$

if and only if for every $\varepsilon>0$ we can find a positive integer $K$ such that for all large enough $m$,

$$
\left|\sum_{k=K}^{N_{m}} Q_{m}\left(f p_{k}\right) q_{k}(\lambda)\right|<\varepsilon .
$$

Proof. For any fixed $J$ and all $m$ large enough,

$$
\begin{aligned}
Q_{m}^{N_{m}}(f ; \lambda)-I(f ; \lambda)= & \sum_{k=0}^{N_{m}} Q_{m}\left(f p_{k}\right) q_{k}(\lambda)-\sum_{k=0}^{\infty}\left(f, p_{k}\right) q_{k}(\lambda) \\
= & \sum_{k=0}^{K-1}\left\{Q_{m}\left(f p_{k}\right)-\left(f, p_{k}\right)\right\} q_{k}(\lambda) \\
& -\sum_{k=K}^{\infty}\left(f, p_{k}\right) q_{k}(\lambda)+\sum_{k=K}^{N_{m}} Q_{m}\left(f p_{k}\right) q_{k}(\lambda)
\end{aligned}
$$


Here, as in the proof of Theorem 6 , the first term in this last right-hand side is $o(1)$ as $m \rightarrow \infty$. Further, given $\varepsilon>0$, we can find a $K$ such that the absolute value of the second term in this last right-hand side is bounded above by $\varepsilon$. Hence for $m$ large enough,

$$
\left|Q_{m}^{N_{m}}(f ; \lambda)-I(f ; \lambda)-\sum_{k=K}^{N_{m}} Q_{m}\left(f p_{k}\right) q_{k}(\lambda)\right|<2 \varepsilon,
$$

which proves the theorem.

Department of Applied Mathematics

Weizmann Institute of Science

P.O. Box 26

Rehovot 76100, Israel

E-mail: maweintr@weizmann.bitnet

Centre for Advanced Computing and Decision Support

C.S.I.R.

P.O. Box 395

Pretoria 0001, Republic of South Africa

1. G. CRiscuolo \& G. MAStroianni, "On the convergence of an interpolatory product rule for evaluating Cauchy principal value integrals," Math. Comp., v. 48, 1987, pp. 725-735.

2. G. Criscuolo \& G. Mastroianni, "A bound for the generalized Jacobi functions of the second kind," Calcolo, v. 24, 1987, pp. 193-198.

3. C. DAGNINO, "Extensions of some results for interpolatory product integration rules to rules not necessarily of interpolatory type," SIAM J. Numer. Anal., v. 23, 1986, pp. 1284-1289.

4. P. J. DAVIS \& P. RABINOWitz, Methods of Numerical Integration, 2nd ed., Academic Press, New York, 1984.

5. G. Freud, Orthogonal Polynomials, Akademiai Kiado/Pergamon Press, Budapest, 1971.

6. G. Freud, "On Markov-Bernstein type inequalities and their applications," J. Approx. Theory, v. 19, 1977, pp. 22-37.

7. A. GERASOULIS, "Piecewise-polynomial quadratures for Cauchy singular integrals," SIAM J. Numer. Anal., v. 23, 1986, pp. 891-902.

8. P. HenriCI, Applied and Computational Complex Analysis, Vol. 3, Wiley, New York, 1986.

9. D. LEVIATAN, "The behaviour of the derivatives of the algebraic polynomials of best approximation," J. Approx. Theory, v. 35, 1982, pp. 169-176.

10. D. S. Lubinsky, A. MATE \& P. NEVAI, "Quadrature sums involving $p$ th powers of polynomials," SIAM J. Math. Anal., v. 18, 1987, pp. 531-544.

11. I. P. NATANSON, Constructive Function Theory, Vol. II (transl. by J. R. Schulenberger), Ungar, New York, 1965.

12. P. Nevai, Orthogonal Polynomials, Mem. Amer. Math. Soc., Vol. 213, Amer. Math. Soc., Providence, R.I., 1979.

13. P. NeVAI, "Mean convergence of Lagrange interpolation. III," Trans. Amer. Math. Soc., v. 282, 1984, pp. 669-698.

14. P. Rabinowitz, The Numerical Evaluation of Cauchy Principal Value Integrals, Symposium on Numerical Mathematics, Durban, 1978, pp. 54-82.

15. P. RABINOWITZ, "Some practical aspects in the numerical evaluation of Cauchy principal value integrals," Internat. J. Comput. Math., v. 20, 1986, pp. 283-298.

16. P. RABINOWITZ, "A stable Gauss-Kronrod algorithm for Cauchy principal value integrals," Comput. Math. Appl., v. 12B, 1986, pp. 1249-1254.

17. A. VAN DER SLUIS \& J. R. ZWEERUS, "An appraisal of some methods for computing Cauchy principal value integrals," in Numerische Integration (G. Hämmerlin, ed.), ISNM 45, Birkhäuser Verlag, Basel, 1979, pp. 264-277. 